\section{Analysis of the Be KLL Auger Transition on Beryllium and Beryllium Oxide by AES}

\author{
Christopher F. Mallinson, ${ }^{\text {a) }}$ James E. Castle, and John F. Watts \\ The Surface Analysis Laboratory, Department of Mechanical Engineering Sciences, \\ University of Surrey, Guildford, GU2 7XH, UK
}

(Received 7 August 2013; accepted 1 October 2013; published 18 November 2013)

As-received beryllium, beryllium scribed in vacuum and beryllium oxide were analysed by Auger Electron Spectroscopy. As-received beryllium was analysed at low and high take off angles. Spectra produced demonstrate the change in the KLL structure with increasing oxygen concentration. Survey spectra as well as high resolution Be KLL and O KLL transitions were collected and are presented. (C) 2013 American Vacuum Society.

[http://dx.doi.org/10.1116/11.20130801]

Keywords: beryllium Be; beryllium oxide BeO; Auger electron spectroscopy AES

PACS: 79.20.Fv, 82.80.Pv, 68.35.B-

Accession \#s: 01269, 01270
Technique: AES
Host Material: \#01269: Be foil;
\#01270: BeO foil
Instrument: Thermo Microlab 350
Major Elements in Spectra: Be, O
Minor Elements in Spectra: O, C
Published Spectra: 11
Spectra in Electronic Record: 20
Spectral Category: reference

\section{INTRODUCTION}

As a continuation of the previous XPS investigation on beryllium, the KLL Auger transition of beryllium has been examined. It is shown herein that the beryllium metal signal is highly attenuated by the thin surface oxide layer, of $\sim 3 \mathrm{~nm}$, due to the low kinetic energy of the transition. Upon removal of this oxide by scribing the metal surface in vacuum a striking increase in the beryllium signal is obtained. Little current literature is available regarding the Auger analysis of beryllium or beryllium compounds. Some of the most recent work has used Auger to monitor the oxidation of beryllium for the investigation of the electronic band structure of beryllium (Ref. 1) and a number of authors in the past have studied the KLL Auger transition in both beryllium (Refs. 2-4) and beryllium oxide (Refs. 1-5).

The earliest work on clean beryllium observed two peaks in the derivative spectrum which were identified as $1 \mathrm{~s} 2 \mathrm{~s} 2 \mathrm{~s}$ and $1 \mathrm{~s} 2 \mathrm{p} 2 \mathrm{p}$ transitions at $92 \mathrm{eV}$ and $104 \mathrm{eV}$ respectively (Ref. 2). Another study confirmed the presence of a large sharp peak at $104.5 \mathrm{eV}$ however no peak was observed at $92 \mathrm{eV}$. In addition to the large peak at $104.5 \mathrm{eV}$ two small peaks at 84 and $60 \mathrm{eV}$ were observed which were believed to be first and second order plasmon losses of the main peak (Ref. 3). Oxidation of the beryllium surface leads to formation of a strong peak at $94 \mathrm{eV}$ and weaker satellites at 85, 77 and $66 \mathrm{eV}$ (Ref. 3). It is concluded that clean beryllium has a large Auger peak at $104.5 \mathrm{eV}$ and beryllium oxide at $94 \mathrm{eV}$. Later work confirmed that no peak is observed at $92 \mathrm{eV}$ on clean, oxide free, beryllium, with the main peak position at $\sim 104 \mathrm{eV}$ (Ref. 4). Beryllium oxide has also been confirmed to show a main peak at $94 \mathrm{eV}$ and three satellites with positions approximately 87, 77 and $67 \mathrm{eV}$ (Ref. 5).

The main peak in $\mathrm{BeO}$ was confirmed to shift by $\sim 10 \mathrm{eV}$ from that of Be (Refs. 1 and 5), the main peak at $95 \mathrm{eV}$ and the satellite at $86 \mathrm{eV}$ originate from the $1 \mathrm{~s} 2 \mathrm{p} 2 \mathrm{p}$ transitions involving electrons from the top and bottom of the p-band respectively. The satellite at $78 \mathrm{eV}$ is believed to be due to the $1 \mathrm{~s} 2 \mathrm{p} 2 \mathrm{~s}$ transition and the satellite at $68 \mathrm{eV}$ is thought to be an energy loss feature (Ref. 5).

a) Author to whom correspondence should be addressed.
In this work spectra are presented that show the main Auger transition for beryllium and beryllium oxide. The beryllium metal differential spectrum shows the primary peak at $108.8 \mathrm{eV}$ together with a loss satellite at $91 \mathrm{eV}$. The native oxide differential spectrum contains the metal component at $108.3 \mathrm{eV}$, the main oxide component at $97 \mathrm{eV}$ as well as a number of satellites at $88.5,81$ and $70 \mathrm{eV}$. The spectrum of native beryllium oxide film was acquired by tilting the sample 15 degrees from the analyser, increasing the emission angle from 60 to 75 degrees relative to the sample normal. The resultant spectrum was free of the metal component. The differential spectrum showed the main oxide component at $97 \mathrm{eV}$ and the satellites at 88.5, 81 and $70 \mathrm{eV}$. The differential spectrum showed the main oxide component at $97 \mathrm{eV}$ and the satellites at 80.5 and $69 \mathrm{eV}$.

To the authors' knowledge this is the first time a sample of bulk beryllium oxide has been analysed. The insulating surface makes analysis difficult. A range of methods were attempted to analyse the surface including: flooding with low energy argon, tilting the sample towards the analyser, covering the sample with aluminium foil and performing analysis through a pin hole in the foil. Each of these methods proved unsuccessful. Analysis using a beam energy of $1.5 \mathrm{kV}$ was partially successful with carbon contamination and oxygen peaks resolvable. The spectrum of beryllium oxide was successfully collected by tilting the sample 85 degrees from the excitation source and reducing the beam energy from 10 to $3 \mathrm{kV}$. Flooding the sample with low energy argon, $<30 \mathrm{eV}$, decreases the small amount of sample charging in the spectrum observed using this method, although it is not removed entirely.

\section{SPECIMEN DESCRIPTION (ACCESSION \#01269, 1 OF 2) -}

Host Material: Be foil

CAS Registry \#: 7440-41-7

Host Material Characteristics: homogeneous; solid; polycrystalline; conductor; metal

Chemical Name: beryllium

Source: GoodFellow

Host Composition: Be

Form: foil $1 \times 25 \times 25 \mathrm{~mm}$

Lot \#: BE000330 
As Received Condition: Sample arrived vaccum packed wrapped in cardboard from GoodFellow. It was stored wrapped in aluminium foil under normal conditions. Typical analysis provided by GoodFellow (ppm) $\mathrm{Al}<500, \mathrm{~B}<3, \mathrm{Cd}$ $<2, \mathrm{Ca}<100, \mathrm{C}<700, \mathrm{Cr}<100, \mathrm{Co}<10, \mathrm{Cu}<100, \mathrm{Fe}<700$, $\mathrm{Pb}<20, \mathrm{Li}<3, \mathrm{Mg}<500, \mathrm{Mn}<120, \mathrm{Mo}<20, \mathrm{Ni}<200, \mathrm{~N}$ $<400, \mathrm{Si}<400, \mathrm{Ag}<10, \mathrm{BeO}<8000$.

Analyzed Region: not specified

Ex Situ Preparation/Mounting: Sample was mounted onto an aluminium sample stub using sprung copper beryllium clips.

In Situ Preparation: Sample was argon ion etched for $60 \mathrm{~s}$ to remove carbonaceous contamination. After initial analysis was completed, a region was scribed with the end of a wobble stick, and the freshly exposed beryllium analysed.

Pre-Analysis Beam Exposure: Sample was previously exposed to approximately $15 \mathrm{~h}$ of monochromatic Al x-ray irradiation and sonically cleaned in isopropyl alcohol for 5 min.

Charge Control: not specified

Temp. During Analysis: $300 \mathrm{~K}$

Pressure During Analysis: $<1 \times 10^{-7} \mathrm{~Pa}$

\section{SPECIMEN DESCRIPTION (ACCESSION \#01270, 2 OF 2) -}

Host Material: $\mathrm{BeO}$ foil

CAS Registry \#: 1304-56-9

Host Material Characteristics: homogeneous; solid; polycrystalline; dielectric; inorganic compound; ceramic

Chemical Name: beryllium oxide

Source: GoodFellow

Host Composition: $\mathrm{BeO}$

Form: foil $1 \times 25 \times 25 \mathrm{~mm}$

Lot \#: 739-203-48

As Received Condition: Sample arrived vaccum packed wrapped in cardboard from GoodFellow. It was stored wrapped in aluminium foil under normal conditions.

Analyzed Region: not specified

Ex Situ Preparation/Mounting: Due to previous fault during analysis the sample had been sputtered with large amounts of magnesium and silver. These were removed by rinsing the sample in aqua regia for $5 \mathrm{~min}$, then neutralised using sodium hydroxide before the sample was rinsed using deionised water. Sample was mounted onto an aluminium sample stub using sprung copper beryllium clips.

In Situ Preparation: The sample was argon ion etched for $180 \mathrm{~s}$ to remove carbon contamination at which point etching was no longer effective.

Pre-Analysis Beam Exposure: Sample was previously exposed to approximately $15 \mathrm{~h}$ of monochromatic $\mathrm{Al} \mathrm{x}$-ray irradiation and sonically cleaned in isopropyl alcohol for $5 \mathrm{~min}$.

Charge Control: Sample was analysed by using a $3 \mathrm{kV}$ beam energy and tilting the sample 85 degrees from the excitation source.

Temp. During Analysis: $300 \mathrm{~K}$

Pressure During Analysis: $<1 \times 10^{-6} \mathrm{~Pa}$
INSTRUMENT DESCRIPTION

Manufacturer and Model: Thermo Microlab 350

Analyzer Type: spherical sector

Detector: multi-channel parallel detector

Number of Detector Elements: 6

INSTRUMENT PARAMETERS COMMON TO ALL SPECTRA -

\section{Spectrometer}

Analyzer Mode: constant retard ratio

Throughput $\left(T=E^{N}\right): \mathrm{N}=$ See comment below

Throughput Comment: The transmission function is calculated from a cubic polynomial fit to a plot of LOG ( Peak Area * $\mathrm{XSF} / \mathrm{PE})$ vs. LOG $(\mathrm{RR}) \mathrm{RR}=\mathrm{KE} / \mathrm{PE}($ Retard Ratio $=$ Kinetic Energy/Pass Energy). XSF is a term to account for the difference in absolute sensitivities of the Ag MNN and Ag 3d peaks.

Excitation Source Window: none

Excitation Source: electron beam

Source Beam Size: $0.1 \mu \mathrm{m} \times 0.1 \mu \mathrm{m}$

Source Beam Size at Specimen Surface: $0.11 \mu \mathrm{m} \times 0.11 \mu \mathrm{m}$

Signal Modulation: none

Signal Mode: pulse single channel

Geometry

Incident Angle: $60^{\circ}$

Source to Analyzer Angle: $60^{\circ}$

Emission Angle: $60^{\circ}$

Specimen Azimuthal Angle: $0^{\circ}$

Acceptance Angle from Analyzer Axis: $50 /+25^{\circ}$

Analyzer Angular Acceptance Width: $25^{\circ} \times 25^{\circ}$

\section{Ion Gun}

Manufacturer and Model: Thermo EX05

Energy: $3000 \mathrm{eV}$

Current: $1 \mathrm{~mA}$

Current Measurement Method: biased stage

Sputtering Species: $\mathrm{Ar}^{+}$

Spot Size (unrastered): $200 \mu \mathrm{m}$

Raster Size: $2000 \mu \mathrm{m} \times 2000 \mu \mathrm{m}$

Incident Angle: $67.5^{\circ}$

Polar Angle: $43^{\circ}$

Azimuthal Angle: $228^{\circ}$

\section{DATA ANALYSIS METHOD}

Energy Scale Correction: The Be KLL, O KLL and survey spectra from beryllium oxide have been shifted to correct for charging. Each is shifted to align the maximum peak intensity with that seen in the equivalent spectra obtained from the tilted beryllium metal.

Recommended Energy Scale Shift: The Be KLL spectra has been shifted by $+6.5 \mathrm{eV}$ and the O KLL and survey spectra have been shifted by $+5 \mathrm{eV}$. 
Peak Shape and Background Method: A Shirley background was used for fitting peaks.

Quantitation Method: Peak height was used to measure peak amplitude and the FWHM of each peak measured using a Shirley background. Peaks were fitted using Thermo Scientific Avantage v3.75 software.

\section{ACKNOWLEDGMENTS}

The Authors wish to thank the EPRSC, the Engineering Doctorate Programme at the University of Surrey and the sponsorship of AWE.

\section{REFERENCES}

1. V. A. Sashin, M. A. Bolorizadeh, A. S. Kheifets, and M. J. Ford, J. Phys.: Condens. Matter 15, 3567-3581 (2003).

2. R. J. Fortner and R. G. Musket, Phys. Rev. Lett. 26, 80-82 (1971).

3. M. Suleman and E. B. Pattinson, J. Phys. F: Met. Phys. 3, 497504 (1971).

4. G. Hanke and K. Muller, Surf. Sci. 152, 902-910 (1985).

5. E. J. Lejeune and R. D. Dixon, J. Appl. Phys. 43, 1998-1999 (1971). 


\begin{tabular}{|c|c|c|c|c|c|c|c|}
\hline \multicolumn{8}{|c|}{ SPECTRAL FEATURES TABLE } \\
\hline $\begin{array}{l}\text { Spectrum } \\
\text { ID \# }\end{array}$ & $\begin{array}{l}\text { Element/ } \\
\text { Transition }\end{array}$ & $\begin{array}{c}\text { Peak Energy } \\
(\mathrm{eV})\end{array}$ & $\begin{array}{l}\text { Peak Width } \\
\text { FWHM (eV) }\end{array}$ & $\begin{array}{l}\text { Peak Area } \\
(\mathrm{eV} \times \mathrm{cts} / \mathrm{s})\end{array}$ & $\begin{array}{l}\text { Sensitivity } \\
\text { Factor }\end{array}$ & $\begin{array}{c}\text { Concentration } \\
\text { (at. \%) }\end{array}$ & Peak Assignment \\
\hline 01269-02 & $\mathrm{Be} \mathrm{KLL}$ & 103.3 & 8.7 & 14726 & $\ldots$ & $\ldots$ & Be metal \\
\hline 01269-09 & O KLL & 510.3 & 10.5 & 1690 & $\ldots$ & $\cdots$ & $\mathrm{O}$ in $\mathrm{BeO}$ \\
\hline 01269-04 & $\mathrm{Be}$ KLL & $102.1,94.5$ & 7.7 & 9324 & $\ldots$ & $\ldots$ & $\mathrm{Be}$ in $\mathrm{BeO}$ and metal \\
\hline 01269-05 & O KLL & 508.2 & 12.3 & 8471 & $\ldots$ & $\cdots$ & $\mathrm{O}$ in $\mathrm{BeO}$ \\
\hline 01269-03 & C KLL & 262.7 & 16.7 & 880 & $\ldots$ & $\cdots$ & C in contamination \\
\hline 01269-07 & Be KLL & 94.5 & 5.9 & 4153 & $\ldots$ & $\ldots$ & $\mathrm{Be}$ in $\mathrm{BeO}$ \\
\hline 01269-08 & O KLL & 506.9 & 8.4 & 11416 & $\ldots$ & $\ldots$ & $\mathrm{O}$ in $\mathrm{BeO}$ \\
\hline 01269-06 & C KLL & 267.8 & 19.0 & 2271 & $\cdots$ & $\cdots$ & C in contamination \\
\hline $01270-02^{\mathrm{a}}$ & $\mathrm{Be} \mathrm{KLL}$ & 86.0 & 8.0 & 49621 & $\ldots$ & $\ldots$ & $\mathrm{Be}$ in $\mathrm{BeO}$ \\
\hline $01270-03^{b}$ & O KLL & 501.0 & 11.4 & 152881 & $\ldots$ & $\ldots$ & $\mathrm{O}$ in $\mathrm{BeO}$ \\
\hline 01270-01 & C KLL & 259.0 & 24.0 & 33896 & $\ldots$ & $\ldots$ & C in contamination \\
\hline 01270-01 & Ar LMM & 209.0 & 5.6 & 7290 & $\ldots$ & $\ldots$ & Implanted argon \\
\hline
\end{tabular}

${ }^{\text {a }}$ Peak energy not adjusted for charging which is believed to be $\sim 6.5 \mathrm{eV}$

${ }^{\mathrm{b}}$ Peak energy not adjusted for charging which is believed to be $\sim 5 \mathrm{eV}$

\begin{tabular}{lccccccc}
\hline \multicolumn{7}{c}{ ANALYZER CALIBRATION TABLE } \\
\hline $\begin{array}{l}\text { Spectrum } \\
\text { ID \# }\end{array}$ & $\begin{array}{c}\text { Element/ } \\
\text { Transition }\end{array}$ & $\begin{array}{c}\text { Peak Energy } \\
(\mathbf{e V})\end{array}$ & $\begin{array}{c}\text { Peak Width } \\
\text { FWHM (eV) })\end{array}$ & $\begin{array}{c}\text { Peak Area } \\
(\mathbf{e V} \times \mathbf{c t s} / \mathbf{s})\end{array}$ & $\begin{array}{c}\text { Sensitivity } \\
\text { Factor }\end{array}$ & $\begin{array}{c}\text { Concentration } \\
\text { (at. \%) }\end{array}$ & $\begin{array}{c}\text { Peak } \\
\text { Assignment }\end{array}$ \\
\hline 15 & $\mathrm{Au} \mathrm{M} \mathrm{M}_{6,7} \mathrm{~N}_{6,7}$ & 2014.0 & 22 & 19676 & $\ldots$ & $\ldots$ & Au metal \\
14 & $\mathrm{Au} \mathrm{N}_{6,7} \mathrm{VV}$ & $69.5,71.5$ & 7.4 & 38605 & $\ldots$ & $\ldots$ & Au metal \\
20 & $\mathrm{Ag} \mathrm{M}_{4} \mathrm{NN}$ & $351.75,357.5$ & 4.4 & 111196 & $\ldots$ & $\ldots$ & Ag metal \\
18 & $\mathrm{Cu} \mathrm{L}_{3} \mathrm{VV}$ & $60.2,62.3$ & 6.2 & 19337 & $\ldots$ & $\ldots$ & Cu metal \\
17 & $\mathrm{Cu} \mathrm{M}_{2,3} \mathrm{VV}$ & 917.3 & 7.2 & 41899 & $\ldots$ & $\ldots$ & Cu metal \\
\hline
\end{tabular}




\begin{tabular}{lccccc}
\hline \multicolumn{2}{c}{ GUIDE TO FIGURES } & & & \\
\hline Spectrum (Accession) \# & Spectral Region & Voltage Shift & Multiplier & Baseline & Comment \# \\
\hline $1269-01$ & Be KLL, O KLL & -5 & 1 & 0 & 1 \\
$1269-02$ & Be KLL & -6.5 & 1 & 0 & 1 \\
$1269-03$ & Be KLL, C KLL, O KLL & -5 & 1 & 0 & 1 \\
$1269-04$ & Be KLL & -6.5 & 1 & 0 & 1 \\
$1269-05$ & O KLL & -5 & 1 & 0 & 1 \\
$1269-06$ & Be KLL, C KLL, O KLL & -5 & 1 & 0 & 1 \\
$1269-07$ & Be KLL & -6.5 & 1 & 0 & 1 \\
$1269-08$ & O KLL & -5 & 1 & 0 & 1 \\
$1270-01$ & Be KLL, C KLL, O KLL & -5 & 1 & 0 & 2 \\
$1270-02$ & Be KLL & -6.5 & 1 & 0 & 2 \\
$1270-03$ & O KLL & -5 & 1 & 0 & 2 \\
$1269-09$ [NP] & O KLL & -5 & 1 & 0 & 1 \\
$1271-01$ [NP] & Au NVV, Au MNN & - & 1 & 0 & 3 \\
$1271-02[N P]$ & Au NVV & - & 1 & 0 & 3 \\
$1271-03[N P]$ & Au MNN & - & 1 & 0 & 3 \\
$1272-01$ [NP] & Cu MVV, Cu LVV & - & 1 & 0 & 4 \\
$1272-02[N P]$ & Cu MVV & - & 1 & 0 & 4 \\
$1272-03[N P]$ & Cu LVV & - & 1 & 0 & 4 \\
$1273-01$ [NP] & Ag MNN & - & 1 & 0 & 5 \\
$1273-02[N P]$ & Ag MNN & - & 1 & 0 & 5 \\
\hline
\end{tabular}

"Voltage shift of the archived (as-measured) spectrum relative to the printed figure. The figure reflects the recommended energy scale correction due to a calibration correction, sample charging, flood gun, or other phenomenon.

${ }^{* *}$ [NP] signifies not published; digital spectra are archived in SSS database but not reproduced in the printed journal.

1. Be

2. $\mathrm{BeO}$

3. Au calibration

4. Cu calibration

5. Ag calibration 


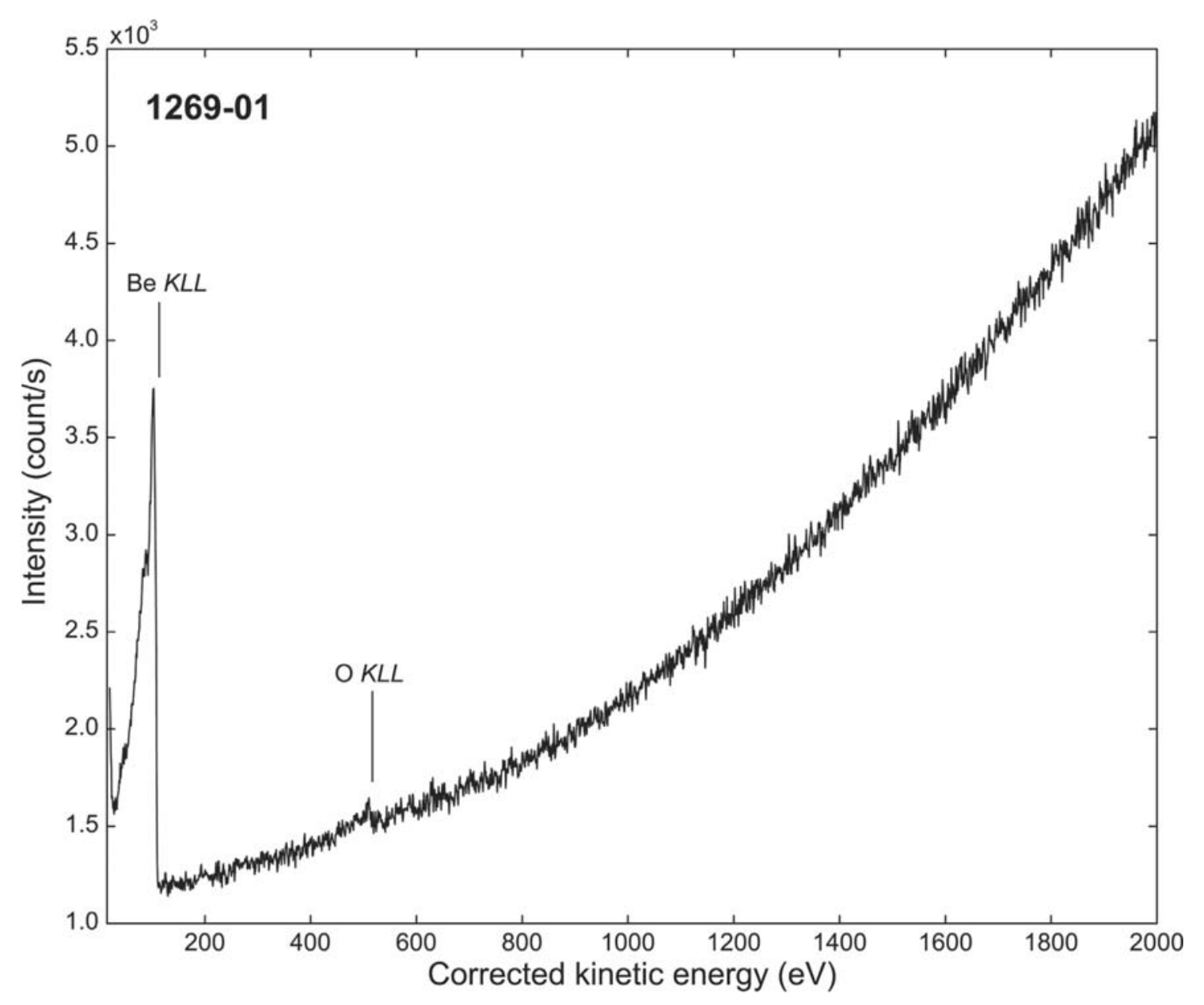

\begin{tabular}{rc}
\hline Accession \# & 01269-01 \\
Host Material & Be foil \\
Technique & AES \\
Spectral Region & survey \\
Instrument & Thermo Microlab 350 \\
Excitation Source & electron beam \\
Source Energy & $10000 \mathrm{eV}$ \\
Source Strength & $1.22 \mathrm{nA}$ \\
Source Size & $0.1 \mu \mathrm{m} \times 0.1 \mu \mathrm{m}$ \\
Analyzer Type & spherical sector \\
Incident Angle & $60^{\circ}$ \\
Emission Angle & $60^{\circ}$ \\
Analyzer Retard Ratio: & 4 \\
Analyzer Resolution & $2 \%$ \\
Total Elapsed Time & $495 \mathrm{~s}$ \\
Number of Scans & $720 \mathrm{~s}$ \\
Total Signal Accumulation Time & 5 \\
Coctive Detector Width & $1 \mathrm{eV}$ \\
\hline
\end{tabular}




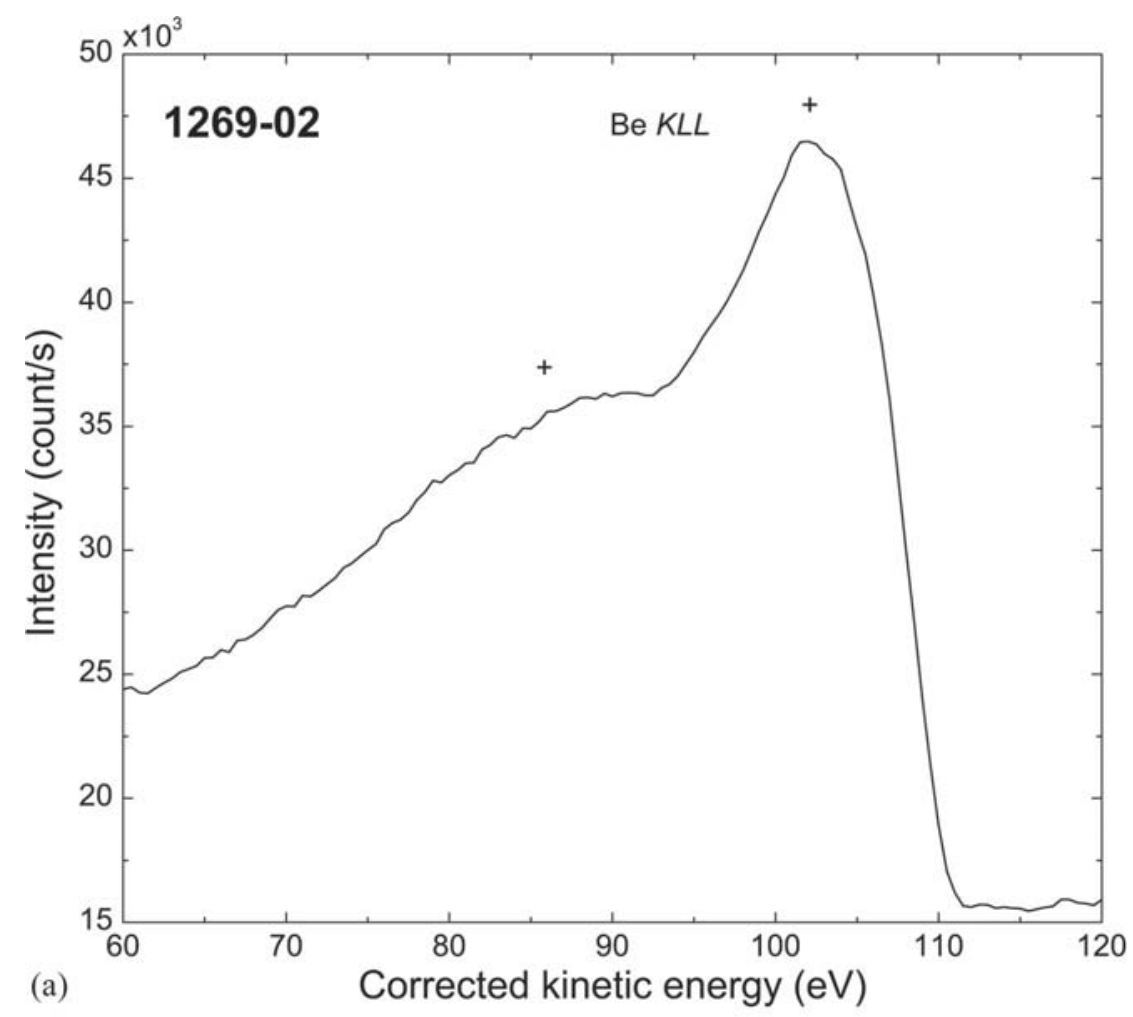

- Accession \#: 01269-02

- Host Material: Be foil

- Technique: AES

- Spectral Region: Be KLL

Instrument: Thermo Microlab 350

Excitation Source: electron beam

Source Energy: $10000 \mathrm{eV}$

Source Strength: $1.22 \mathrm{nA}$

Source Size: $0.1 \mu \mathrm{m} \times 0.1 \mu \mathrm{m}$

Analyzer Type: spherical sector

Incident Angle: $60^{\circ}$

Emission Angle: $60^{\circ}$

Analyzer Retard Ratio: 4

Analyzer Resolution: $2 \%$

Total Signal Accumulation Time: $604 \mathrm{~s}$

Total Elapsed Time: $900 \mathrm{~s}$

Number of Scans: 100

Effective Detector Width: $0.5 \mathrm{eV}$

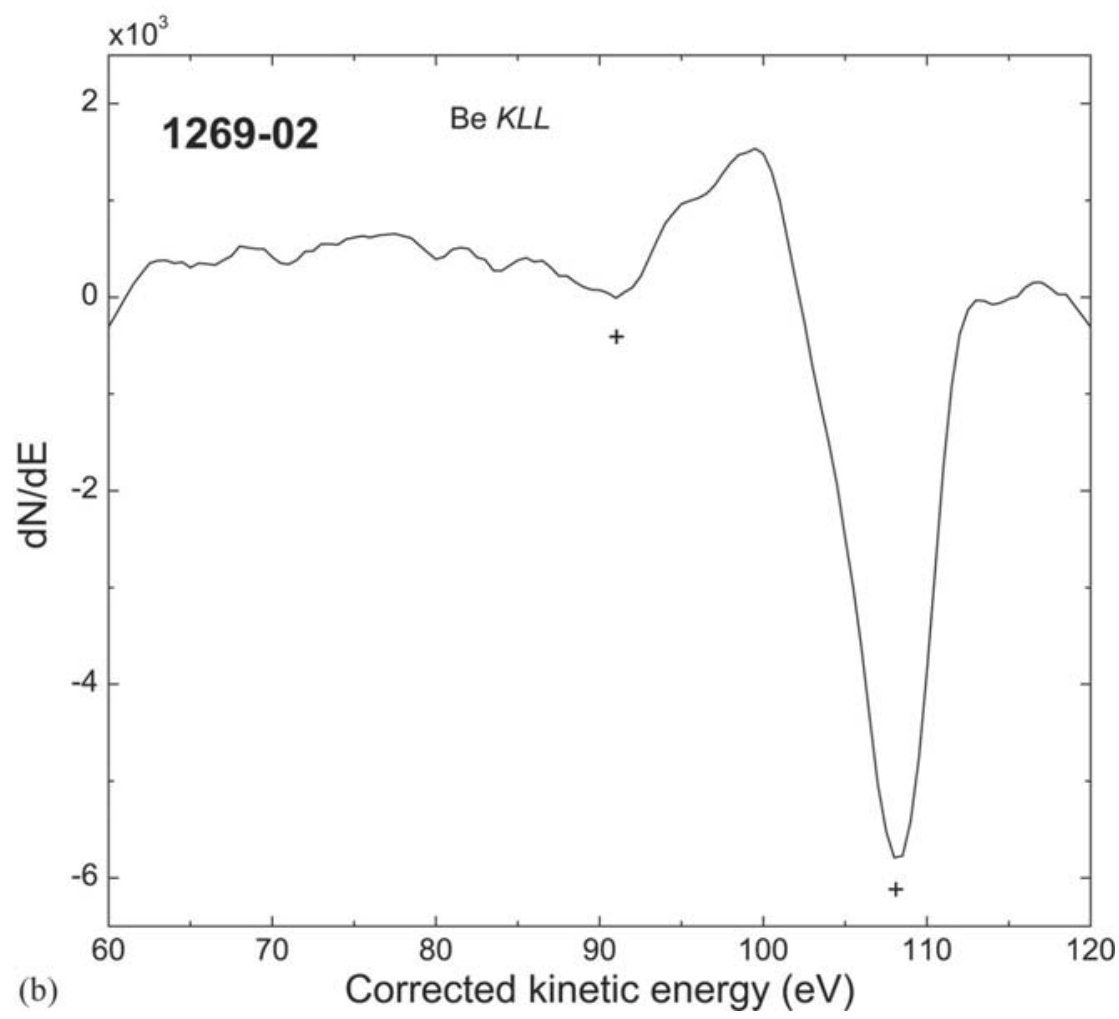




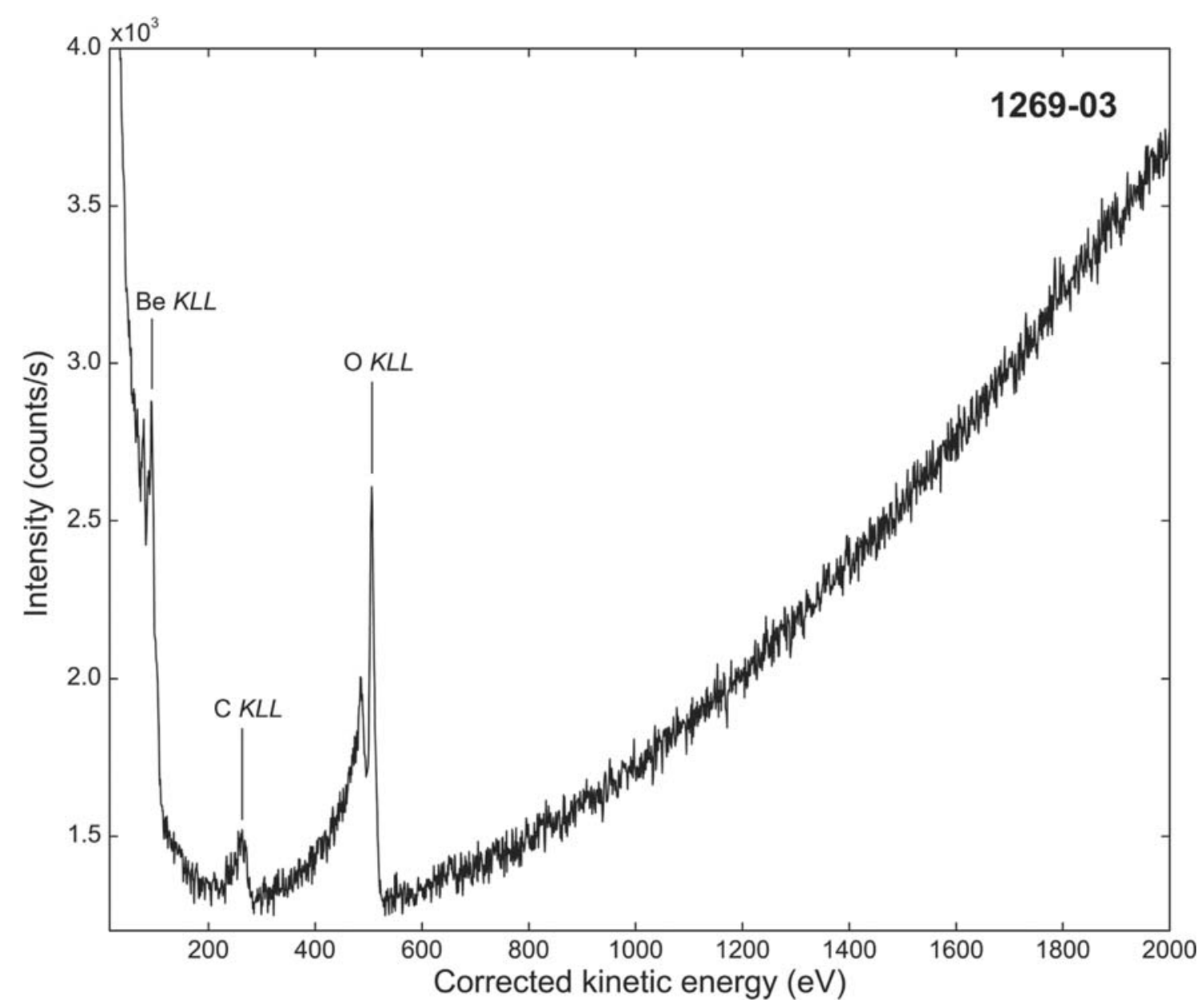

\begin{tabular}{|c|c|}
\hline Accession \# & 01269-03 \\
\hline Host Material & Be foil \\
\hline Technique & AES \\
\hline Spectral Region & survey \\
\hline Instrument & Thermo Microlab 350 \\
\hline Excitation Source & electron beam \\
\hline Source Energy & $10000 \mathrm{eV}$ \\
\hline Source Strength & $1.22 \mathrm{nA}$ \\
\hline Source Size & $0.1 \mu \mathrm{m} \times 0.1 \mu \mathrm{m}$ \\
\hline Analyzer Type & spherical sector \\
\hline Incident Angle & $60^{\circ}$ \\
\hline Emission Angle & $60^{\circ}$ \\
\hline Analyzer Retard Ratio: & 4 \\
\hline Analyzer Resolution & $2 \%$ \\
\hline Total Signal Accumulation Time & $495 \mathrm{~s}$ \\
\hline Total Elapsed Time & $727 \mathrm{~s}$ \\
\hline Number of Scans & 5 \\
\hline Effective Detector Width & $1 \mathrm{eV}$ \\
\hline Comment & $\begin{array}{l}\text { Survey of as-received metal surface showing } \\
\text { both oxide and metal Be KLL components }\end{array}$ \\
\hline
\end{tabular}




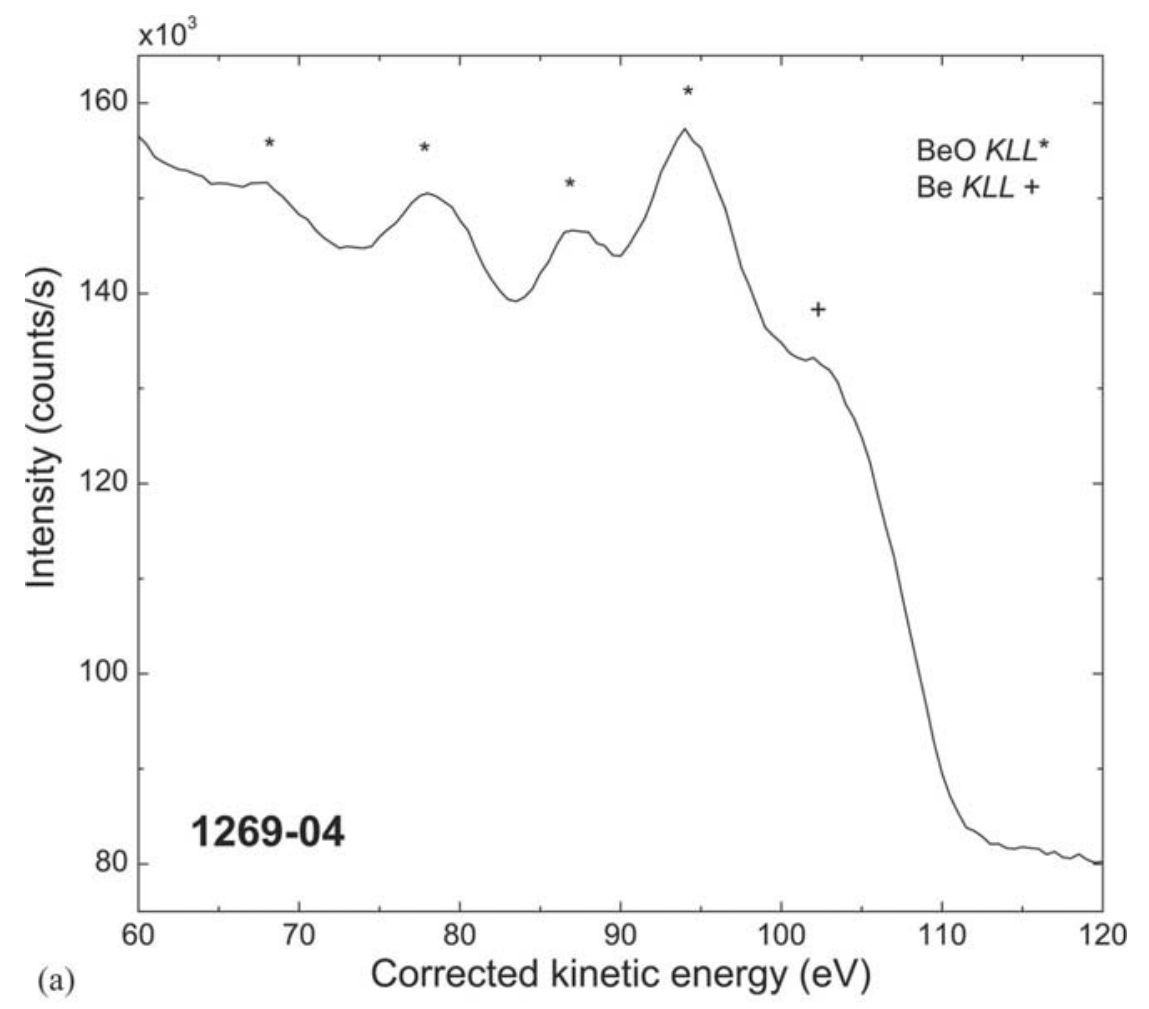

- Accession \#: 01269-04

- Host Material: Be foil

- Technique: AES

- Spectral Region: Be KLL

Instrument: Thermo Microlab 350

Excitation Source: electron beam

Source Energy: $10000 \mathrm{eV}$

Source Strength: $1.22 \mathrm{nA}$

Source Size: $0.1 \mu \mathrm{m} \times 0.1 \mu \mathrm{m}$

Analyzer Type: spherical sector

Incident Angle: $60^{\circ}$

Emission Angle: $60^{\circ}$

Analyzer Retard Ratio: 4

Analyzer Resolution: $2 \%$

Total Signal Accumulation Time: $465 \mathrm{~s}$

Total Elapsed Time: $555 \mathrm{~s}$

Number of Scans: 50

Effective Detector Width: $0.5 \mathrm{eV}$

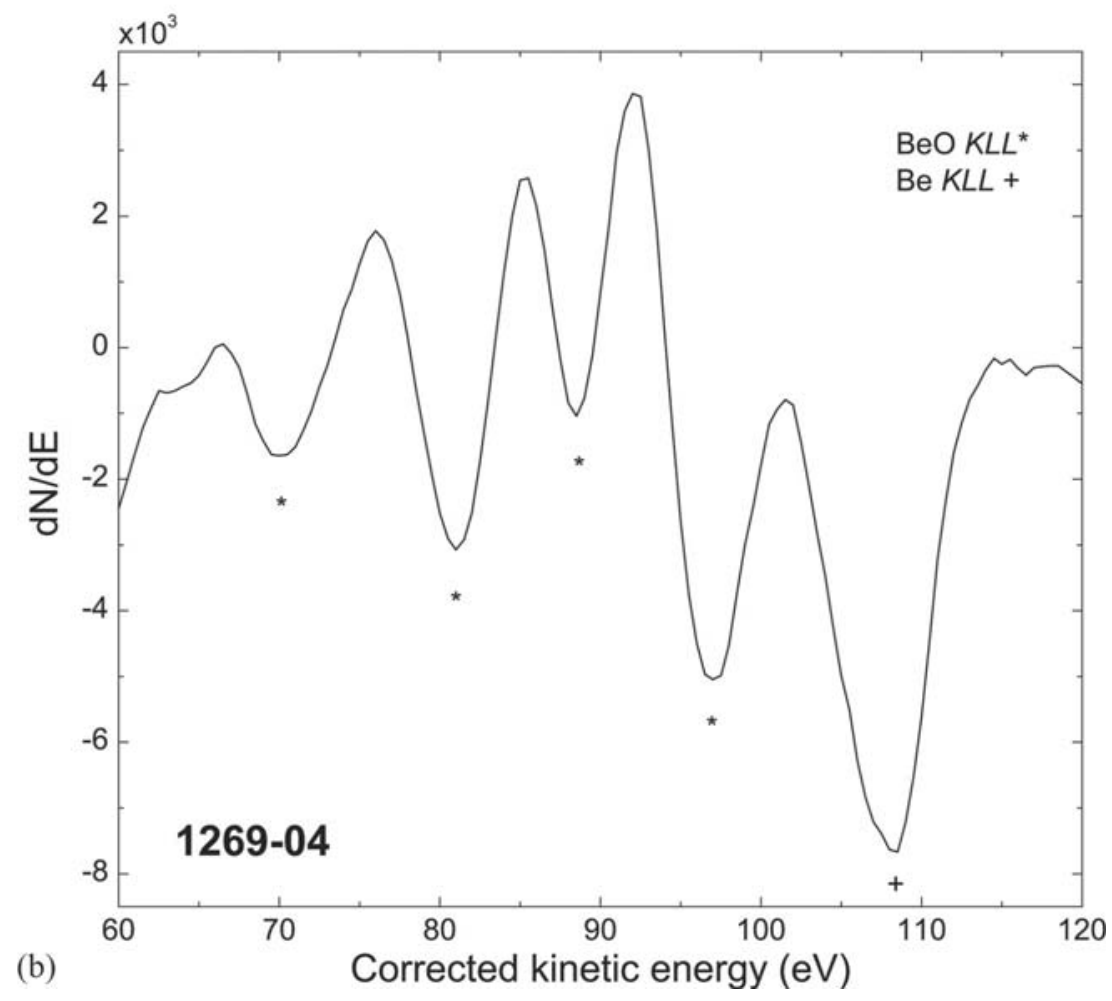




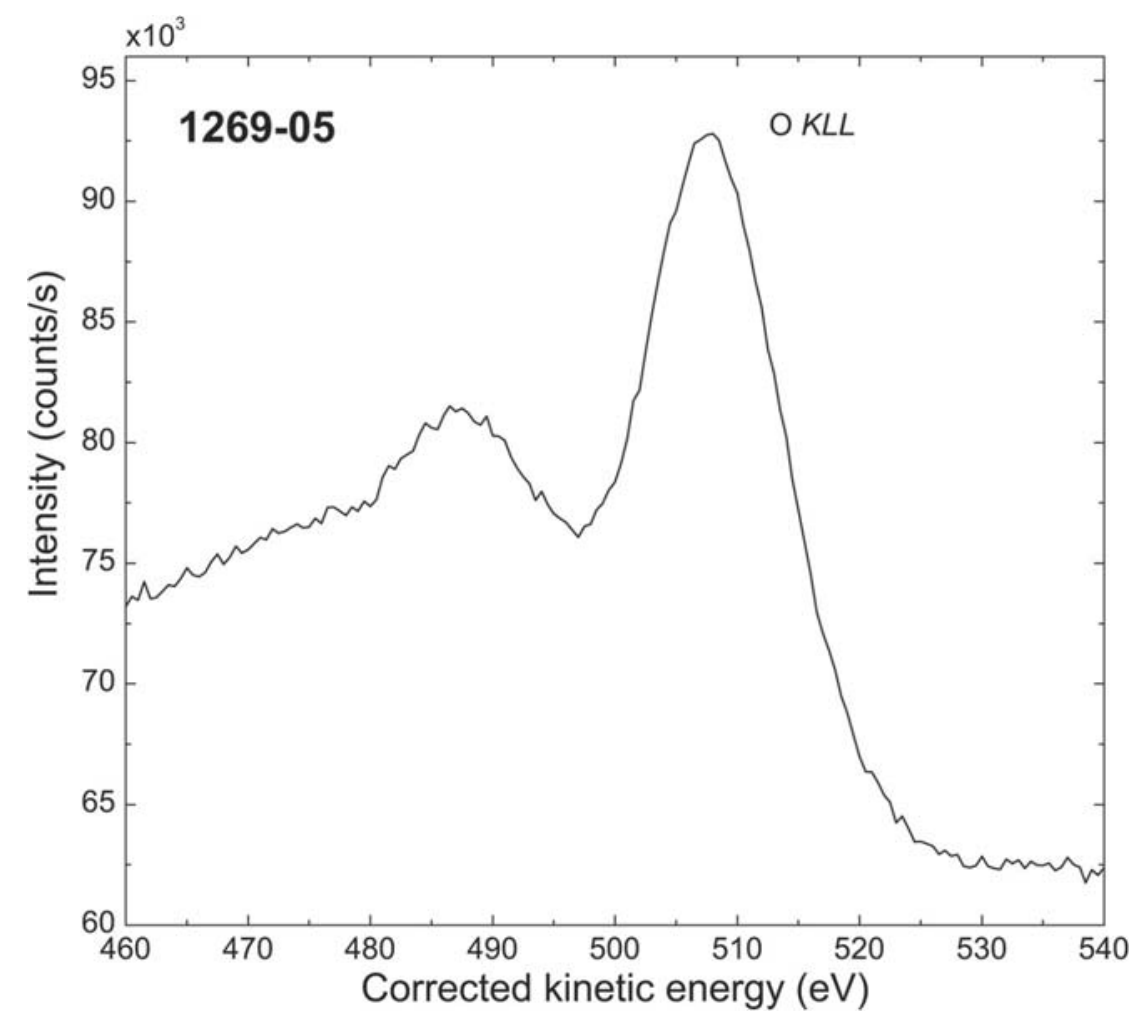

- Accession \#: 01269-05

- Host Material: Be foil

- Technique: $A E S$

- Spectral Region: O KLL

Instrument: Thermo Microlab 350

Excitation Source: electron beam

Source Energy: $10000 \mathrm{eV}$

Source Strength: $1.22 \mathrm{nA}$

Source Size: $0.1 \mu \mathrm{m} \times 0.1 \mu \mathrm{m}$

Analyzer Type: spherical sector

Incident Angle: $60^{\circ}$

Emission Angle: $60^{\circ}$

Analyzer Retard Ratio: 4

Analyzer Resolution: $2 \%$

Total Signal Accumulation Time: 402 s

Total Elapsed Time: $925 \mathrm{~s}$

Number of Scans: 50

Effective Detector Width: $0.5 \mathrm{eV}$ 


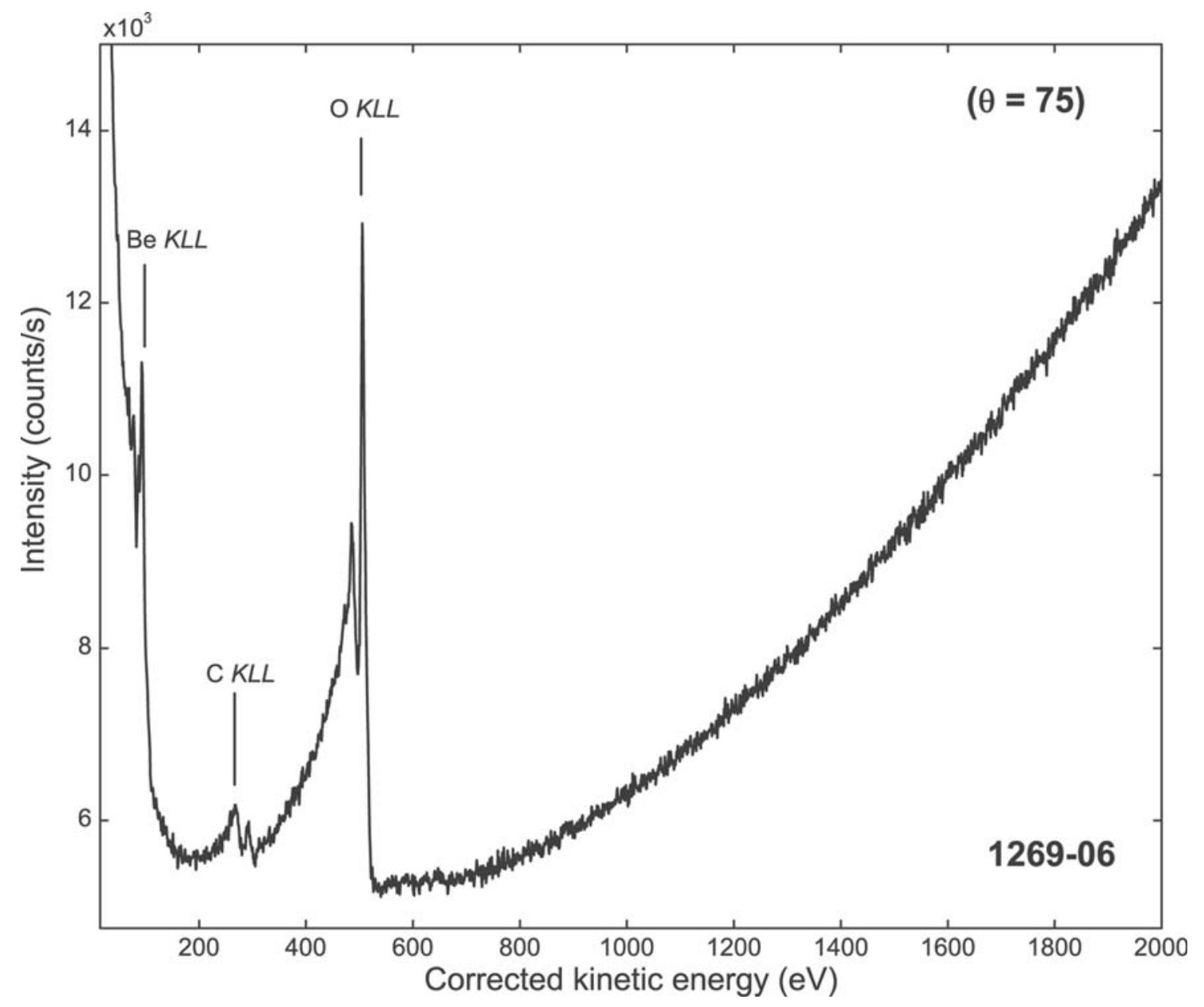

\begin{tabular}{rc}
\hline Accession \# & $01269-06$ \\
\hline Host Material & Be foil \\
Technique & AES \\
Spectral Region & survey \\
Instrument & Thermo Microlab 350 \\
Excitation Source & electron beam \\
Source Energy & $10000 \mathrm{eV}$ \\
Source Strength & $1.22 \mathrm{nA}$ \\
Source Size & $0.1 \mu \mathrm{m} \times 0.1 \mu \mathrm{m}$ \\
Analyzer Type & spherical sector \\
Incident Angle & $60^{\circ}$ \\
Emission Angle & $75^{\circ}$ \\
Analyzer Retard Ratio: & 4 \\
Analyzer Resolution & $2 \%$ \\
Total Elapsed Time & $495 \mathrm{~s}$ \\
Number of Scans & $710 \mathrm{~s}$ \\
Total Signal Accumulation Time & 5 \\
Effective Detector Width & $1 \mathrm{eV}$ \\
Comment & to isolate surface oxide and collect just BeO KLL component
\end{tabular}




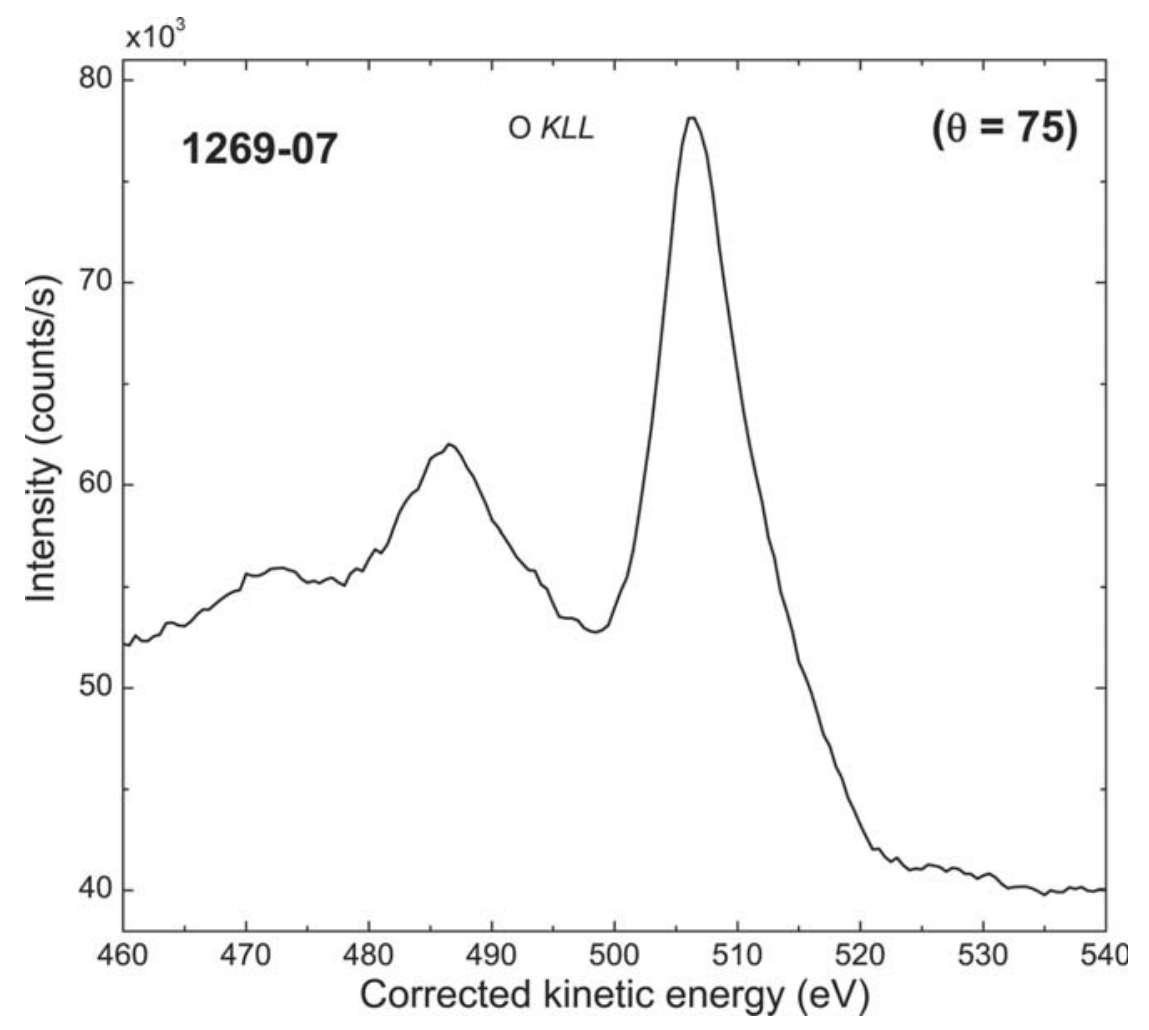

- Accession \#: 01269-07

- Host Material: Be foil

- Technique: AES

- Spectral Region: Be KLL

Instrument: Thermo Microlab 350

Excitation Source: electron beam

Source Energy: $10000 \mathrm{eV}$

Source Strength: $1.22 \mathrm{nA}$

Source Size: $0.1 \mu \mathrm{m} \times 0.1 \mu \mathrm{m}$

Analyzer Type: spherical sector

Incident Angle: $60^{\circ}$

Emission Angle: $75^{\circ}$

Analyzer Retard Ratio: 4

Analyzer Resolution: $2 \%$

Total Signal Accumulation Time: $604 \mathrm{~s}$

Total Elapsed Time: $866 \mathrm{~s}$

Number of Scans: 100

Effective Detector Width: $0.5 \mathrm{eV}$ 


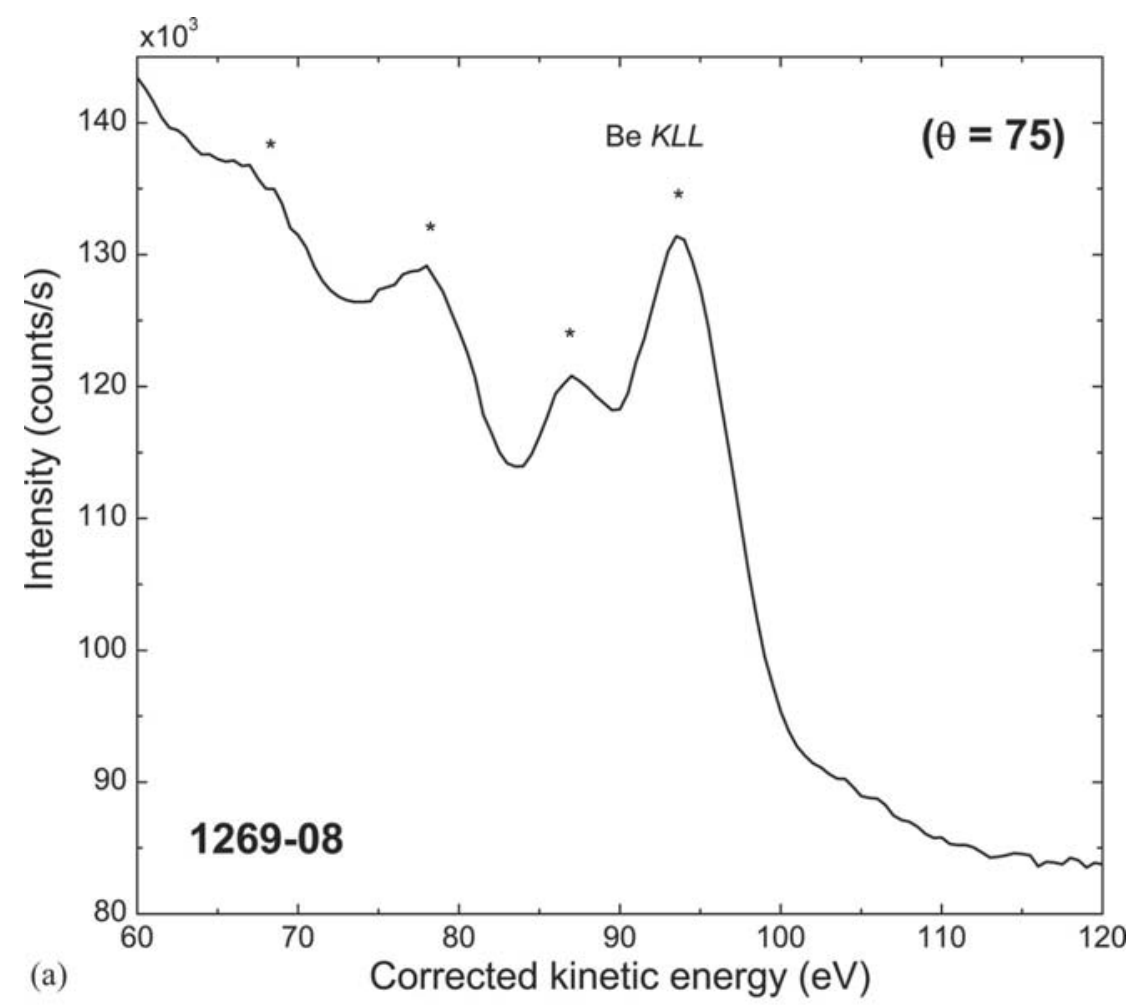

Accession \#: 01269-08

- Host Material: Be foil

- Technique: AES

- Spectral Region: O KLL

Instrument: Thermo Microlab 350

Excitation Source: electron beam

Source Energy: $10000 \mathrm{eV}$

Source Strength: $1.22 \mathrm{nA}$

Source Size: $0.1 \mu \mathrm{m} \times 0.1 \mu \mathrm{m}$

Analyzer Type: spherical sector

Incident Angle: $60^{\circ}$

Emission Angle: $75^{\circ}$

Analyzer Retard Ratio: 4

Analyzer Resolution: $2 \%$

Total Signal Accumulation Time: $402 \mathrm{~s}$

Total Elapsed Time: $648 \mathrm{~s}$

Number of Scans: 50

Effective Detector Width: $0.5 \mathrm{eV}$

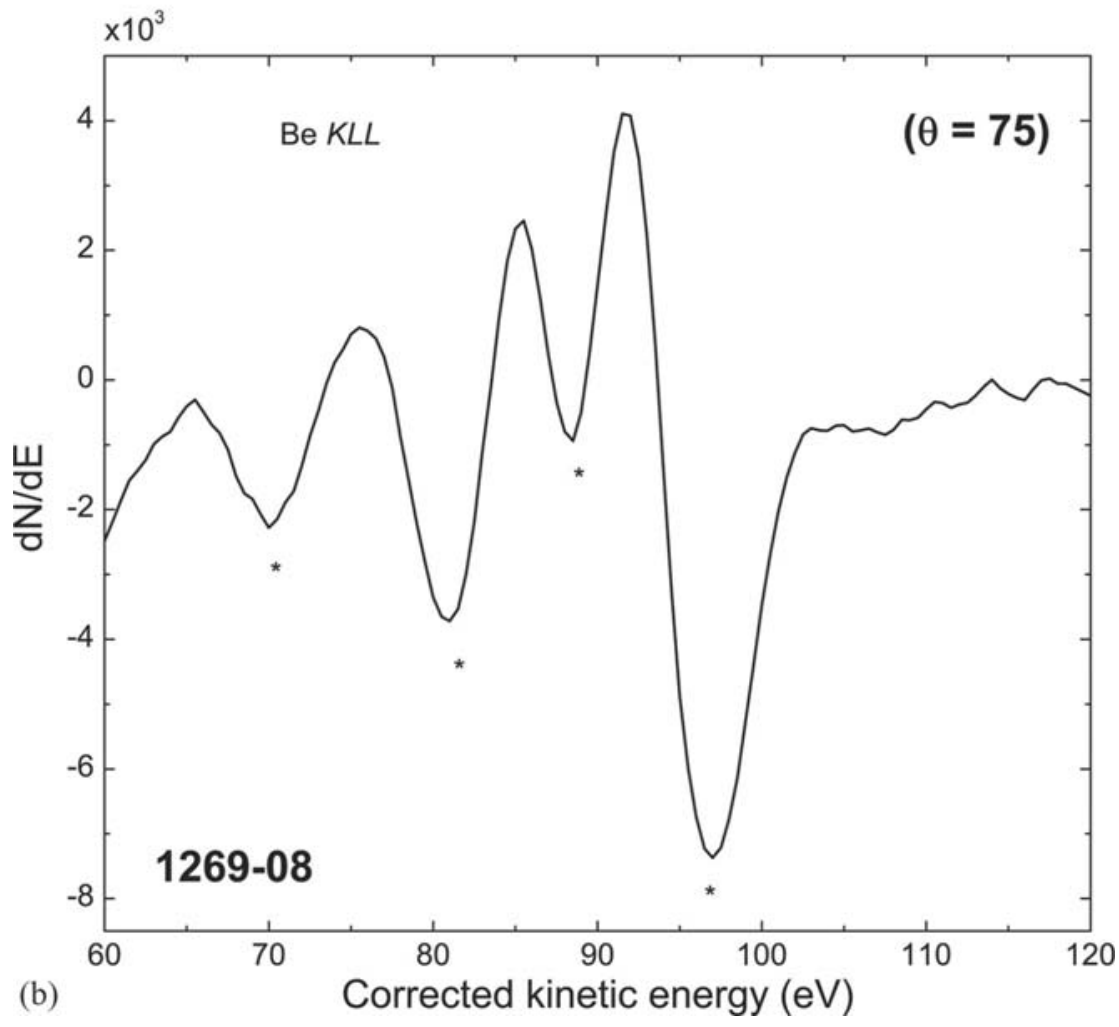




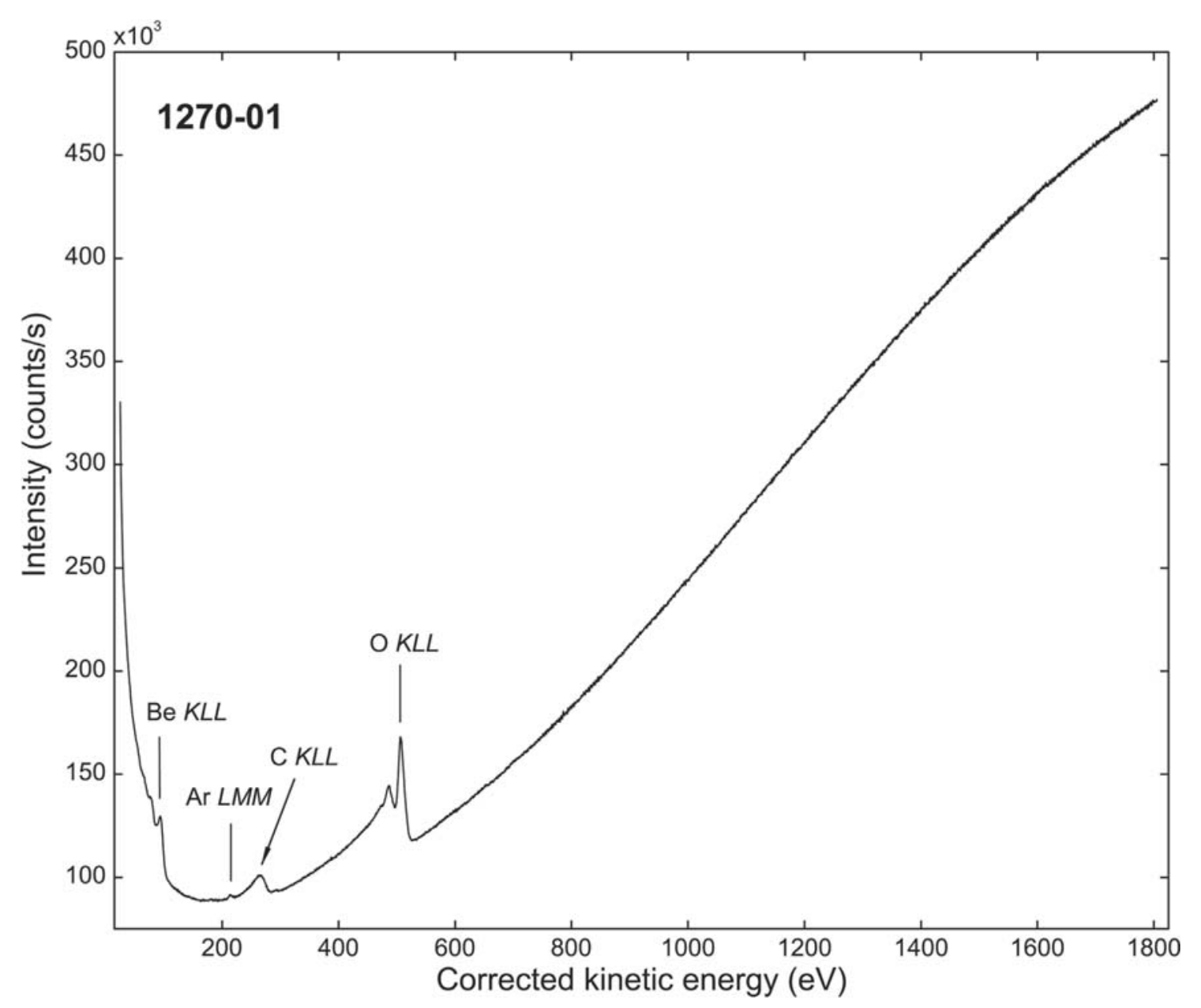

\begin{tabular}{rc}
\hline Accession \# & 01270-01 \\
\hline Host Material & BeO foil \\
Technique & AES \\
Spectral Region & survey \\
Instrument & Thermo Microlab 350 \\
Excitation Source & electron beam \\
Source Energy & $3000 \mathrm{eV}$ \\
Source Strength & $0.04 \mathrm{nA}$ \\
Source Size & $0.1 \mu \mathrm{m} \times 0.1 \mu \mathrm{m}$ \\
Analyzer Type & spherical sector \\
Incident Angle & $60^{\circ}$ \\
Emission Angle & $60^{\circ}$ \\
Analyzer Retard Ratio: & 4 \\
Analyzer Resolution & $2 \%$ \\
Total Elapsed Time & $899 \mathrm{~s}$ \\
Number of Scans & $1217 \mathrm{~s}$ \\
Total Signal Accumulation Time & 5 \\
Cffective Detector Width & $0.5 \mathrm{eV}$ \\
Comment & Survey collected from bulk BeO \\
\hline
\end{tabular}




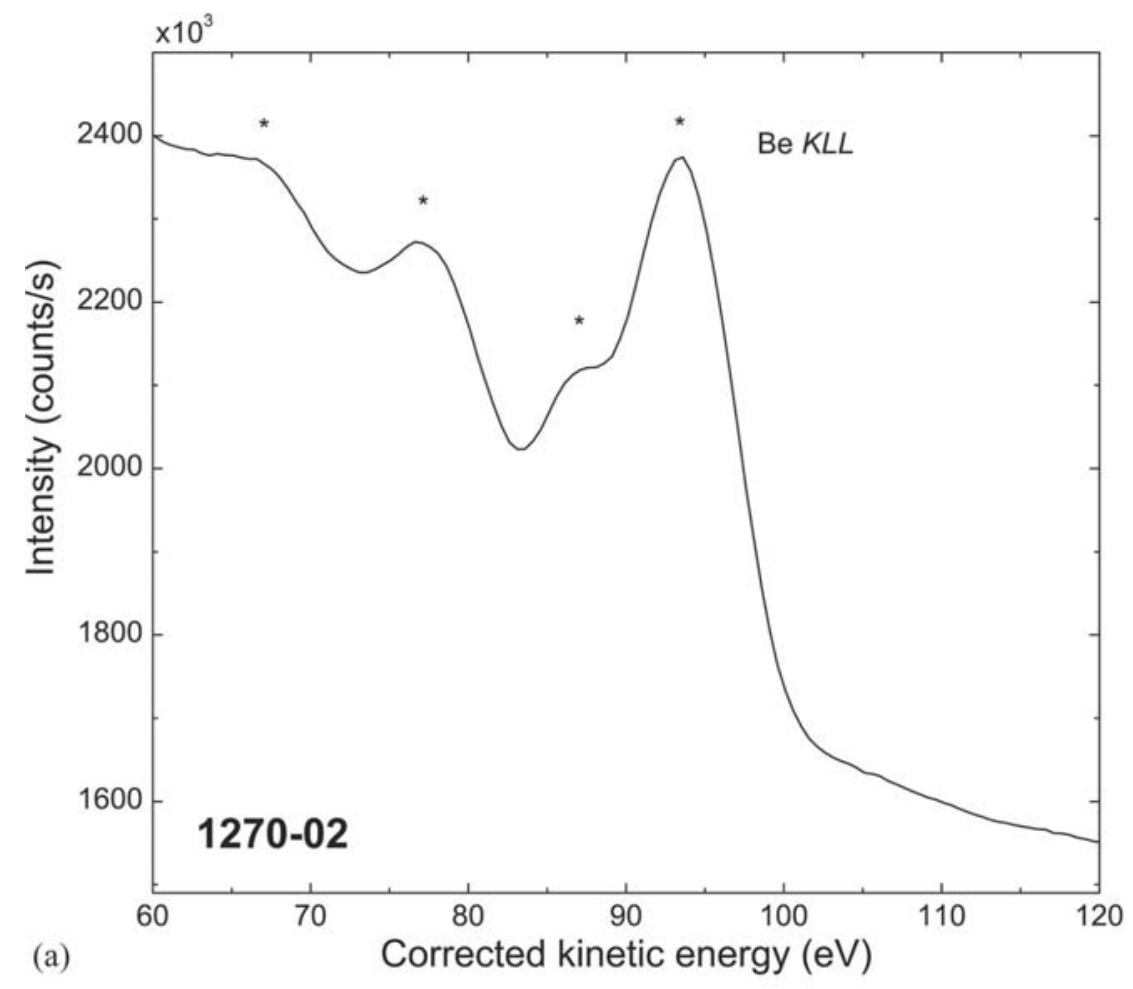

- Accession \#: 01270-02

- Host Material: $\mathrm{BeO}$ foil

- Technique: AES

- Spectral Region: Be KLL

Instrument: Thermo Microlab 350

Excitation Source: electron beam

Source Energy: 3000 eV

Source Strength: $0.04 \mathrm{nA}$

Source Size: $0.1 \mu \mathrm{m} \times 0.1 \mu \mathrm{m}$

Analyzer Type: spherical sector

Incident Angle: $60^{\circ}$

Emission Angle: $60^{\circ}$

Analyzer Retard Ratio: 4

Analyzer Resolution: 2\%

Total Signal Accumulation Time: $376 \mathrm{~s}$

Total Elapsed Time: $552 \mathrm{~s}$

Number of Scans: 50

Effective Detector Width: $0.5 \mathrm{eV}$

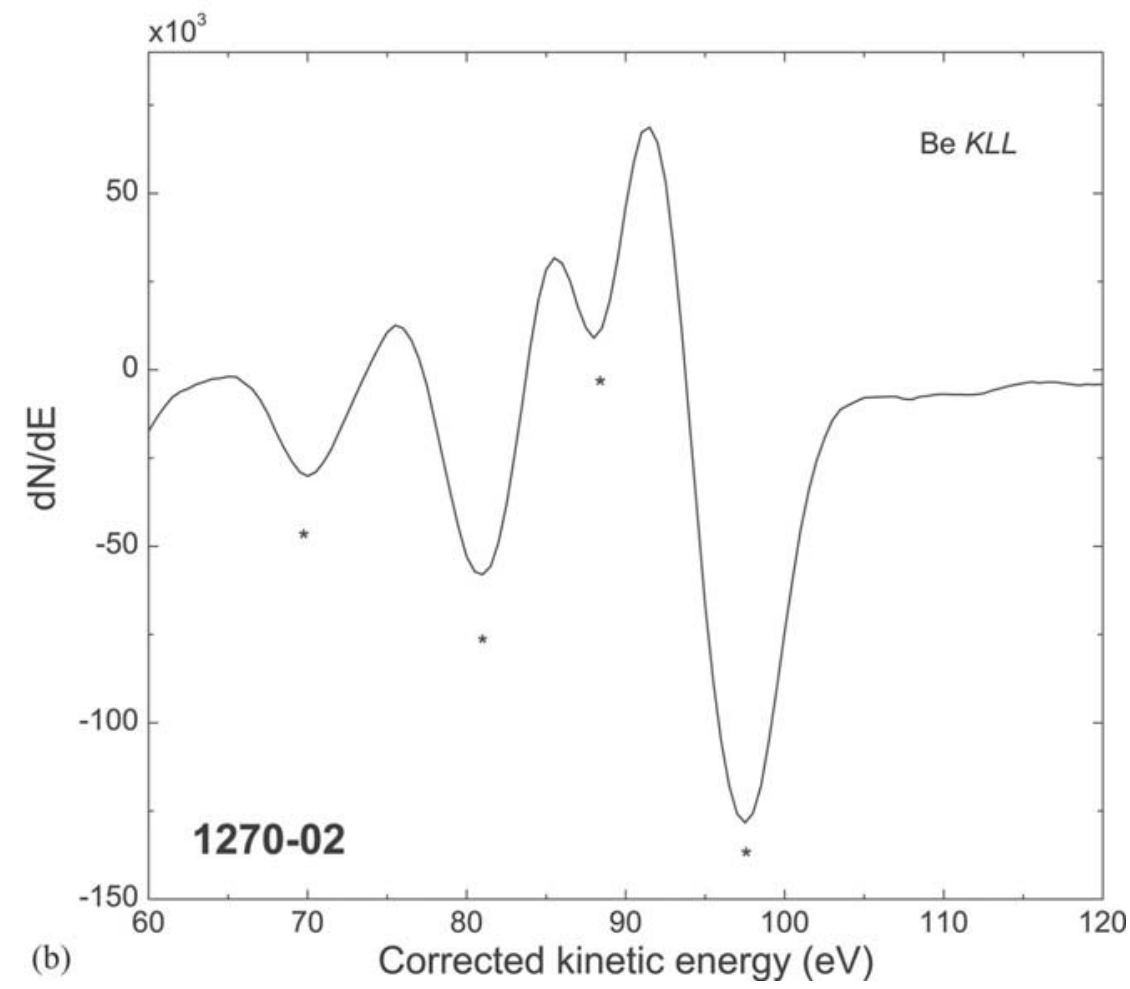




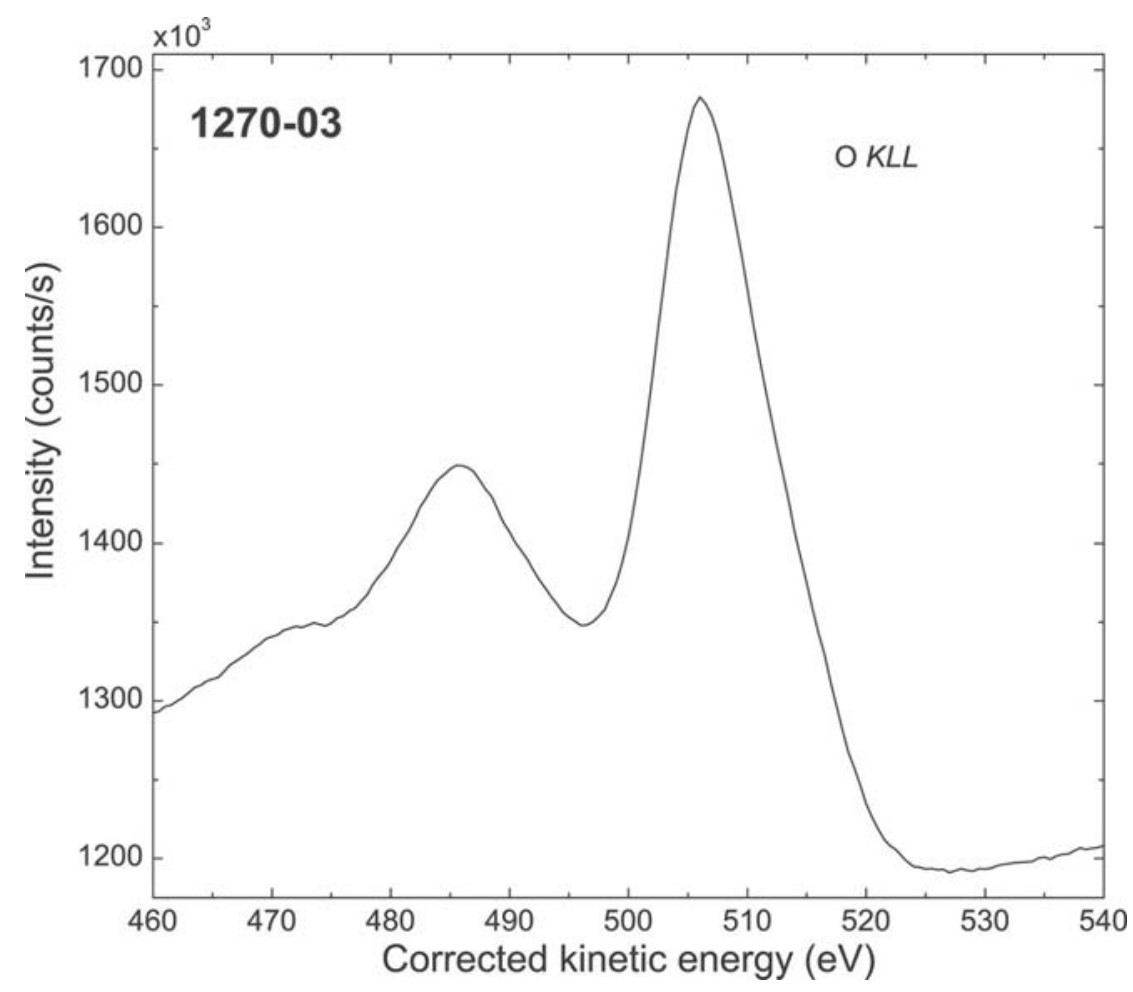

- Accession \#: 01270-03

- Host Material: $\mathrm{BeO}$ foil

- Technique: AES

- Spectral Region: O KLL

Instrument: Thermo Microlab 350

Excitation Source: electron beam

Source Energy: $3000 \mathrm{eV}$

Source Strength: $0.04 \mathrm{nA}$

Source Size: $0.1 \mu \mathrm{m} \times 0.1 \mu \mathrm{m}$

Analyzer Type: spherical sector

Incident Angle: $60^{\circ}$

Emission Angle: $60^{\circ}$

Analyzer Retard Ratio: 4

Analyzer Resolution: $2 \%$

Total Signal Accumulation Time: $502 \mathrm{~s}$

Total Elapsed Time: $790 \mathrm{~s}$

Number of Scans: 50

Effective Detector Width: $0.5 \mathrm{eV}$ 